\title{
Sonolência Diurna e Habilidades Sociais em Estudantes de Medicina
}

\author{
Daytime Sleepiness and Social Skills Among \\ Medical Students
}

\author{
Maria Cristina Kloster \\ Bruno Perotta \\ Alfredo Hauer Junior \\ Helena BMS Paro ${ }^{I I}$ \\ Patrícia Tempski
}

\section{PALAVRAS-CHAVE: \\ - Inteligência Social; \\ - Educação Médica; \\ - Distúrbios do Sono por Sonolência Excessiva;}

\section{KEYWORDS:}

- Social Intelligence;

- Medical Education;

- Disorders of Excessive

Somnolence.

Recebido em: 05/12/2011

Reencaminhado em: 16/04/2012

Reencaminhado em: 19/09/2012

Aprovado em: 26/02/2013

REVISTA BRASILEIRA DE EDUCAÇ̄̃o MÉDICA

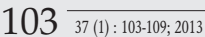

${ }_{\text {I Faculdade de Medicina Evangélica do Paraná, Curitiba, PR, Brasil. }}$
${ }^{\text {II }}$ Universidade Federal de Uberlândia, Uberlândia, MG, Brasil.

\section{RESUMO}

A prática médica depende de um repertório de competências sociais que permitem ao médico desenvolver empatia, habilidades de comunicação, pensamento crítico, capacidade de liderança e tomada de decisões. Nosso objetivo foi analisar as habilidades sociais e a presença de sonolência diurna entre estudantes de Medicina nos quatro primeiros anos do curso. Os estudantes $(n=180)$ responderam ao Inventário de Habilidades Sociais de Del Prette E Del Prette e à Escala de Sonolência Diurna. Observou-se que não houve diferença dos escores de habilidades sociais nos diferentes anos do curso e que $47,3 \%$ dos estudantes apresentaram escores baixos, necessitando de aprimoramento. Estudantes do sexo feminino apresentaram maior autocontrole da agressividade. Entre os estudantes, 50\% apresentaram sonolência diurna, cuja presença se associou a menores escores de habilidades sociais, principalmente do fator relativo à autoexposição a desconhecidos ou a situações novas.

The practice of medicine requires social skills enabling a doctor to develop empathy, communication skills, critical thinking, leadership skills and the ability to make a decision. Our objective was to evaluate medical students' social skills and the latter's relationship with daytime sleepiness. Students (n=180) responded to the Del Prette \& Del Prette Social Skills Questionnaire and the Epworth Sleepiness Scale. We observed that scores for social skills did not differ according to the student's current year of their medical course and that $47.3 \%$ of students presented low scores indicating the need for developing these skills. Women were more able to control aggressiveness. 50\% of students recorded pathological scores for daytime sleepiness and this was associated with lower scores for social skills, especially concerning exposure to strangers or new situations. 


\section{INTRODUÇÃO}

O ensino médico no Brasil é norteado pelas Diretrizes Curriculares Nacionais (DCN), que definem os princípios, fundamentos, condições e procedimentos da formação de médicos, estabelecidos pela Câmara de Educação Superior do Conselho Nacional de Educação. Segundo as DCN, espera-se que ao final do curso de graduação em Medicina o egresso seja um profissional com formação generalista, humanista, crítica e reflexiva, capacitado a atuar no processo de saúde-doença em seus diferentes níveis de atenção, pautado em princípios éticos. O médico deve adotar ações de promoção, prevenção, recuperação e reabilitação à saúde, na perspectiva da integralidade da assistência, com senso de responsabilidade social e compromisso com a cidadania, como promotor da saúde integral do ser humano.

As DCN definem as competências gerais do médico: atenção à saúde, que envolve pensar criticamente, analisar problemas, procurar soluções; tomada de decisões, que engloba habilidades de avaliar, sistematizar e decidir as condutas mais adequadas; comunicação verbal e não verbal; liderança, demonstrada pela aptidão para assumir cargos com compromisso e empatia, de forma eficaz e efetiva; administração e gerenciamento e educação permanente ${ }^{1}$.

Essas aptidões dependem do desenvolvimento de uma série de habilidades sociais, que envolvem uma classe de comportamentos existentes no repertório do próprio indivíduo, compondo um desempenho socialmente competente ${ }^{2}$. São exemplos de habilidades sociais: habilidades de comunicação, civilidade, assertivas de direito e cidadania, empatia, expressão de sentimento positivo e habilidade para o trabalho ${ }^{3}$

Estudos recentes, no entanto, mostram que a vivência do curso de Medicina nem sempre privilegia o desenvolvimento das competências gerais das DCN e das habilidades sociais requeridas por elas ${ }^{4,5,6}$.

Durante o curso de Medicina, o estudante vivencia momentos de crise. A adaptação necessária no momento de ingresso no curso, o contato com o processo saúde-doença e seus determinantes sociais, o excesso de conteúdo teórico e prático a ser assimilado, o aprendizado das diversas habilidades, atitudes e valores éticos da profissão médica e o processo de consolidação da identidade médica podem ser fatores que contribuem para esses momentos de adversidade ${ }^{7,8}$. Alguns estudantes superam essas adversidades com maior tranquilidade, aprendem e se desenvolvem com elas, buscando auxílio psicológico quando necessário, enquanto outros podem desenvolver quadros psicopatológicos ${ }^{4,9}$.
De fato, vários autores observam que o curso médico não é bom para a saúde e qualidade de vida do aluno. A possível associação entre saúde e bem-estar dos estudantes com a qualidade do cuidado e desempenho acadêmico tem estimulado estudos na área da educação médica. Tem-se observado que a maioria dos estudantes de Medicina apresenta escores patológicos de sonolência diurna ${ }^{10,11,12} \mathrm{e}$ que estudantes com maior sonolência apresentam pior qualidade de vida ${ }^{10}$ e desempenho acadêmico ${ }^{13}$. É possível que a sonolência diurna excessiva observada entre os estudantes de Medicina esteja associada a um pior desempenho das habilidades sociais.

O presente estudo tem por objetivo avaliar os escores de habilidades sociais dos estudantes de Medicina e sua associação com a presença de sonolência diurna nas fases do curso que antecedem o internato médico.

\section{MÉTODO}

Trata-se de um estudo transversal, que utilizou uma amostra de conveniência, composta por 167 acadêmicos de Medicina de uma instituição de ensino privada em Curitiba, sendo 48 alunos matriculados no primeiro ano, 46 alunos no segundo, 35 alunos no terceiro e 38 alunos no quarto ano. O estudo foi aprovado pelo Comitê de Ética e Pesquisa, e a participação dos estudantes foi voluntária e condicionada à assinatura de termo de consentimento livre esclarecido. Como parte da ética da pesquisa, o estudo ofereceu garantia de acesso a suporte psicopedagógico aos participantes por meio do Grupo de Apoio Multidisciplinar ao Aluno (Gama).

\section{Instrumentos de medida}

Os estudantes receberam um caderno de resposta autoinforme não identificado com os seguintes instrumentos: dados sociodemográficos, Inventário de Habilidades Sociais (IHS) ${ }^{14}$ e Escala de Sonolência Diurna de Epworth ${ }^{15}$. A aplicação do caderno de resposta ocorreu em sala de aula durante o horário letivo autorizado pela coordenação do curso, no meio do semestre letivo, longe do período de férias ou provas e após o término das atividades didáticas

\section{Inventário de Habilidades Sociais (IHS)}

A avaliação do desenvolvimento das habilidades sociais foi proposta por Del Prette \& Del Prette, que elaboraram e validaram um Inventário de Habilidades Sociais (IHS). O inventário é composto por um escore total e por cinco esco- 
res fatoriais: assertividade, autoestima, comunicação, autoexposição e autocontrole da agressividade. A interpretação dos valores pode também ser feita por percentis. Escores entre os percentis zero e 50 indicam a necessidade de treinamento em habilidades sociais. Escores entre os percentis 50 e 100 correspondem a um desempenho adequado nessas habilidades ${ }^{14}$.

\section{Escala de Sonolência Diurna de Epworth}

Elaborada e validada por Johns Muray15, a escala contém oito afirmações com respostas de 0 a 3 e escores que variam de 0 a 24 e foi desenvolvida para avaliar a tendência à sonolência em situações cotidianas. É um instrumento autoaplicável, validado para uso no Brasil. Escores maiores do que 10 indicam sonolência diurna excessiva e são também chamados escores patológicos, e quando acima de 16 são considerados muito patológicos ${ }^{15}$.

\section{Análise estatística}

A avaliação da interdependência entre variáveis categóricas foi realizada pelo teste de qui-quadrado. Para comparação das médias entre dois e três ou mais grupos, foi usado o teste $t$ de Student e análise de variância, respectivamente. A condição de homogeneidade entre as variâncias foi avaliada pelo teste de Levene. Um valor de p inferior a 0,05 foi considerado estatisticamente significativo. O programa estatístico utilizado foi o Statistica, versão 5.1.

Foram excluídos os sujeitos com dados incompletos, ou seja, optou-se por incluir na análise somente os sujeitos com a totalidade das respostas válidas.

\section{RESULTADOS}

Do total de 233 estudantes matriculados do primeiro ao quarto ano do curso médico, foram analisados 180 estudantes, e 167 responderam completamente ao IHS, havendo, assim, perda de 13 respondentes. Noventa alunos eram do sexo feminino $(53,8 \%)$ e 77 do masculino $(46,2 \%)$, com idade variando entre 17 e 28 anos (média e desvio padrão $=20,9 \pm 2,0$ anos). A análise dos escores gerais do IHS indicou que 79 estudantes $(47,3 \%)$ se encontravam entre os percentis $0-50$, ou seja, com desempenho considerado inadequado das suas habilidades sociais. Dos estudantes com baixo desempenho nas habilidades sociais, 38 eram do sexo masculino $(49,4 \%)$ e 41 do sexo feminino $(45,6 \%)(p=0,6244)$ (Tabela 1$)$. Não houve diferença estatisticamente significativa nos diferentes anos do curso em relação à presença ou ausência de baixos escores do IHS ( $\mathrm{p}=$ 0,9770) (Tabela 1).
TABELA 1

Distribuição de frequência do Inventário de Habilidades Sociais (IHS) de acordo com a faixa de percentil $(n=167)$

\begin{tabular}{lccccc}
\hline & \multicolumn{2}{l}{ Percentil $\leq \mathbf{5 0}$} & \multicolumn{2}{l}{ Percentil $>50$} & $p^{*}$ \\
\hline Geral & 79 & $(47,3 \%)$ & 88 & $(52,7 \%)$ & \\
Sexo & & & & & 0,6244 \\
Masculino & 38 & $(49,4 \%)$ & 39 & $(50,6 \%)$ & \\
Feminino & 41 & $(45,6 \%)$ & 49 & $(54,4 \%)$ & \\
Ano do curso & & & & & 0,9770 \\
Primeiro & 23 & $(47,9 \%)$ & 25 & $(52,1 \%)$ & \\
Segundo & 21 & $(45,7 \%)$ & 25 & $(54,3 \%)$ & \\
Terceiro & 16 & $(45,7 \%)$ & 19 & $(54,3 \%)$ & \\
Quarto & 19 & $(50,0 \%)$ & 19 & $(50,0 \%)$ & \\
\hline
\end{tabular}

* teste qui-quadrado

Quando analisados os fatores do IHS, o número de estudantes com escores entre os percentis 0-50 do IHS foi o seguinte: $76(45,5 \%)$ no Fator 1 (assertividade); 80 (47,9\%), no Fator 2 (autoestima e autoafirmação); 54 (32,3\%) no Fator 3 (comunicação e desenvoltura social); 63 (37,7\%) no Fator 4 (autoexposição a desconhecidos ou situações novas); 63 (37,7\%) no Fator 5 (autocontrole da agressividade)

Com relação à comparação dos fatores entre os sexos, uma diferença estatisticamente significativa foi demonstrada apenas no Fator 5 (autocontrole da agressividade). Na amostra feminina, apenas 23 das estudantes $(25,6 \%)$ tiveram escores baixos nesse fator, enquanto 40 estudantes do sexo masculino $(51,9 \%)$ tiveram escores do IHS entre os percentis $0-50(p<$ 0,001) (Tabela 2).

Quanto à avaliação da sonolência diurna, 171 estudantes responderam completamente à Escala de Sonolência Diurna de Epworth, havendo uma perda de nove respondentes. A média dos escores foi de 10,6 (DP $=3,8)$, e 84 estudantes $(49,1 \%)$ apresentaram escores patológicos de sonolência diurna (entre 11 e 24). Destes, 19 (11,1\%) estudantes apresentaram escores muito patológicos (entre 16 e 24). Não foi encontrada diferença significativa nos escores da escala de Epworth de acordo com o sexo $(p=0,4521)$ e com o ano do curso ( $p=$ 0,2122) (Tabela 3). 
TABELA 2

Distribuição de frequência dos cinco fatores do Inventário de Habilidades Sociais (IHS) de acordo com a faixa de percentil $(n=167)$

\begin{tabular}{|c|c|c|c|}
\hline Fator IHS & Percentil $\leq 50$ & Percentil > 50 & $p^{*}$ \\
\hline Fator $1^{+}$ & $76(45,5 \%)$ & $91(54,5 \%)$ & \\
\hline Sexo & & & 0,5416 \\
\hline Masculino & $37(48,1 \%)$ & $40(51,9 \%)$ & \\
\hline Feminino & $39(43,3 \%)$ & $51(56,7 \%)$ & \\
\hline Fator $2^{+}$ & $80(47,9 \%)$ & $87(52,1 \%)$ & \\
\hline Sexo & & & 0,1120 \\
\hline Masculino & $42(54,5 \%)$ & $35(45,5 \%)$ & \\
\hline Feminino & $38(42,2 \%)$ & $52(57,8 \%)$ & \\
\hline Fator $3^{+}$ & $54(32,3 \%)$ & $113(67,7 \%)$ & \\
\hline Sexo & & & 0,7656 \\
\hline Masculino & $24(31,2 \%)$ & $53(68,8 \%)$ & \\
\hline Feminino & $30(33,3 \%)$ & $60(66,7 \%)$ & \\
\hline Fator $4^{+}$ & $63(37,7 \%)$ & $104(62,3 \%)$ & \\
\hline Sexo & & & 0,1948 \\
\hline Masculino & $25(32,5 \%)$ & $52(67,5 \%)$ & \\
\hline Feminino & $38(42,2 \%)$ & $52(57,8 \%)$ & \\
\hline Fator $5^{+}$ & $63(37,7$ \%) & $104(62,3 \%)$ & \\
\hline Sexo & & & $<0,001$ \\
\hline Masculino & $40(51,9 \%)$ & $37(48,1 \%)$ & \\
\hline Feminino & $23(25,6 \%)$ & $67(74,4 \%)$ & \\
\hline
\end{tabular}

* teste qui-quadrado

${ }^{\dagger}$ Fator 1: assertividade; Fator 2: autoestima e autoafirmação; Fator 3: comunicação e desenvoltura social; Fator 4: autoexposição a desconhecidos ou situações novas; Fator 5: autocontrole da agressividade

A comparação das médias dos escores do IHS de acordo com a presença de sonolência diurna demonstrou menores escores do IHS (escore geral e Fator 4, referente à autoexposição) entre os estudantes com escores patológicos de sonolência diurna ( $p=0,0354$ e 0,0290, respectivamente) (Tabela 4).
TABELA 3

Média e desvio padrão dos escores da Escala de Sonolência

Diurna de Epworth

\begin{tabular}{lccc}
\hline & N & $\begin{array}{c}\text { Média } \pm \text { desvio } \\
\text { padrão }\end{array}$ & $p$ \\
\hline Geral & 171 & $10,60 \pm 3,77$ & $0,4521^{*}$ \\
Sexo & & & \\
Masculino & 48 & $10,35 \pm 3,84$ & \\
Feminino & 93 & $10,80 \pm 3,71$ & $0,2122^{+}$ \\
Ano do curso & & & \\
Primeiro & 49 & $10,22 \pm 3,80$ & \\
Segundo & 47 & $10,32 \pm 3,61$ & \\
Terceiro & 35 & $11,80 \pm 3,48$ & \\
Quarto & 40 & $10,33 \pm 4.08$ & \\
\hline
\end{tabular}

* teste t de Student; ${ }^{+}$análise de variância

\section{TABELA 4}

Média e desvio padrão dos escores do Inventário de Habilidades Sociais (IHS) de acordo com a faixa de escore da escala de Epworth $(\mathrm{n}=165)$

\begin{tabular}{lccc}
\hline \multirow{2}{*}{ Escores } & \multicolumn{2}{c}{ Escore de Epworth } & \multirow{2}{*}{$*^{*}$} \\
\cline { 2 - 3 } & $\mathbf{0 - 1 0}(\mathbf{N}=\mathbf{8 3})$ & $\mathbf{1 1 - 2 4} \mathbf{( N = 8 2 )}$ & \\
\hline do IHS & $\begin{array}{c}\text { (média } \pm \text { desvio } \\
\text { padrão) }\end{array}$ & $\begin{array}{c}\text { (média } \pm \text { desvio } \\
\text { padrão) }\end{array}$ & \\
Geral & $2,50 \pm 0,41$ & $2,38 \pm 0,40$ & $0,0354^{*}$ \\
Fator $1^{+}$ & $2,22 \pm 0,62$ & $2,09 \pm 0,54$ & 0,1074 \\
Fator $2^{+}$ & $3,04 \pm 0,59$ & $3,00 \pm 0,64$ & 0,6835 \\
Fator $3^{+}$ & $2,64 \pm 0,63$ & $2,51 \pm 0,65$ & 0,2783 \\
${\text { Fator } 4^{+}}^{2,65 \pm 0,83}$ & $2,39 \pm 0,77$ & $0,0290^{*}$ \\
${\text { Fator } 5^{+}}^{2,76 \pm 0,67}$ & $2,64 \pm 0,66$ & 0,2784 \\
\hline
\end{tabular}

* teste t de Student

${ }^{+}$Fator 1: assertividade; Fator 2: autoestima e autoafirmação; Fator 3: comunicação e desenvoltura social; Fator 4: Autoexposição a desconhecidos ou situações novas; Fator 5: autocontrole da agressividade

\section{DISCUSSÃO}

As habilidades sociais requeridas na prática em saúde estão, muitas vezes, restritas à capacidade intuitiva dos profissionais por não serem sistematicamente abordadas na graduação. No entanto, tais habilidades podem ser adquiridas e aprimoradas 
por meios adequados de aprendizagem ${ }^{3}$. Os saberes dessa forma contemplados auxiliam o profissional de saúde na percepção do ambiente, na ampliação de sua capacidade de escuta, percepção e síntese. Neste sentido, um aspecto importante ao se considerar uma formação orientada para a integralidade da assistência e humanização é a inclusão das habilidades sociais como objetivos pedagógicos na graduação ${ }^{16,17}$.

Os dados analisados neste estudo permitem afirmar que metade dos estudantes avaliados apresenta algum déficit de competência em habilidades sociais e que não há variação de escores nos diferentes anos do curso. Ou seja, muito pouco foi agregado ao estudante pelo currículo, seja ele formal, paralelo ou oculto. Caballo ${ }^{2}$ afirma em seus estudos que, nas profissões da área da saúde, maior ênfase é dada à aquisição de competência técnica em detrimento das competências sociais e interpessoais. Por essa razão, é comum encontrar profissionais com excelente competência técnica e que não interagem eficientemente porque não possuem a habilidade social necessária ${ }^{2}$.

Outro ponto a considerar na avaliação das habilidades sociais entre os estudantes de Medicina é o fenômeno de "feminilização" da profissão médica ${ }^{18}$. Nos últimos anos, o número de mulheres matriculadas na escola médica tem sido igual e, em alguns casos, superior ao do número de homens. Tendo em vista características femininas de cuidado, acolhida e escuta, seria plausível esperar maiores escores de habilidades sociais neste grupo. No entanto, os dados desse estudo demonstraram que somente no fator de autocontrole da agressividade houve diferença significativa entre os grupos feminino e masculino.

Alguns autores descrevem um comportamento diferenciado nas mulheres médicas moldado pelo ambiente de ensino e de trabalho ${ }^{19,20}$, já que a "sociogênese representa um vetor de construção do repertório de comportamento" ${ }^{\prime 21}$. Culturalmente, a mulher sempre construiu uma postura de contenção diante das frustrações ${ }^{22}$. Infere-se, desse modo, que o melhor desempenho alcançado pelas mulheres no fator de autocontrole da agressividade do IHS não tenha sido influenciado pelo curso, mas que essa diferença decorra de fatores culturais.

Uma análise pormenorizada de cada fator do IHS é necessária para a compreensão dos resultados deste estudo. $\mathrm{O}$ Fator 1 (assertividade) envolve a expressão de pensamentos e crenças de maneira direta e apropriada, desde que não viole o direito das outras pessoas ${ }^{14}$. Nesse fator, os achados deste estudo permitem afirmar que $45,5 \%$ dos estudantes necessitam de aprimoramento em assertividade e enfrentamento de situações críticas ou de risco.

O desenvolvimento da autoafirmação na expressão de afeto positivo e autoestima (Fator 2) traz como consequência a habilidade de expressar a solidariedade e capacidade de cultivar o afeto nas relações interpessoais ${ }^{14}$. Nesse fator, os achados deste estudo permitem afirmar que $47,9 \%$ dos alunos necessitam desenvolvimento no que tange à autoafirmação de afeto positivo e autoestima. Outro aspecto importante relacionado ao Fator 2 para a formação médica é a subclasse relacionada à empatia ${ }^{23}$, entendida como a habilidade de perceber a experiência subjetiva do outro, compreender o sentimento do outro e o porquê desse sentimento, além de expressar essa compreensão. Pode ser considerada uma virtude e uma habilidade a ser apreendida e aprimorada durante a vida. A empatia é um componente importante do profissionalismo médico ${ }^{17}$.

O Fator 3 refere-se à a comunicação como mecanismo de formação de redes sociais, englobando habilidades de iniciar e encerrar conversação, fazer e responder perguntas, elogiar, dar e receber feedback ${ }^{14}$, competências esperadas do egresso, segundo as $\mathrm{DCN}^{1}$. Essas habilidades permitem ao estudante um adequado relacionamento médico-paciente, um melhor entendimento das reações da pessoa que adoece e de seus familiares frente à doença e o desenvolvimento da capacidade profissional para atuar como efetivo agente de promoção da saúde ${ }^{16,24}$. A análise do Fator 3 mostrou que 32,3\% dos alunos necessitam de treinamento em comunicação e desenvoltura social. As habilidades contempladas pelo Fator 3 contribuem muito para uma prática médica mais eficaz e humanizada, pois os conceitos de saúde e de humanização do atendimento implicam uma relação dialógica no processo de cuidado ${ }^{16,25,26,27}$ e na visão biopsicossocial do indivíduo ${ }^{4,28}$.

O Fator 4 refere-se à autoexposição a desconhecidos ou situações novas. Os resultados deste estudo demonstraram que $37,7 \%$ dos alunos necessitam desenvolver essa habilidade. No que se refere às habilidades relacionadas ao Fator 5, que envolvem a capacidade de reagir a estimulações aversivas de agressão, pilhéria ou descontrole, com razoável controle da raiva e da agressividade $\mathrm{e}^{14}$, o presente estudo demonstrou que $37,7 \%$ dos alunos não sabem expressar esses sentimentos de forma socialmente competente. Evidenciou-se também que, no grupo masculino, uma proporção maior de estudantes apresentou baixo desempenho em relação ao grupo feminino, o que implicaria maior necessidade de desenvolver estas habilidades no sexo masculino.

Outra variável analisada neste estudo e que possivelmente está associada ao desempenho das habilidades sociais entre os estudantes de Medicina é a sonolência. A sonolência diurna está associada a diversas causas, como privação do sono, mudanças do ciclo circadiano, uso de medicações, obstrução de vias aéreas superiores e idade. Dentre essas, a privação do sono é a mais comum entre adultos jovens, e as mudanças do 
ciclo circadiano podem fazer parte da vida do estudante de Medicina ${ }^{11}$. Tempski ${ }^{4}$ descreveu o impacto da formação acadêmica sobre a qualidade de vida e sonolência do estudante de Medicina e demonstrou que a sonolência diurna está associada a pior qualidade de vida. Em concordância com esse dado, Skinner ${ }^{29}$ afirma que a qualidade do sono é fundamental para uma boa qualidade de vida. Os escores de sonolência diurna encontrados neste estudo evidenciaram que quase a metade dos estudantes apresenta sonolência diurna. Esses resultados são semelhantes aos índices encontrados no estudo com 800 alunos do curso de Medicina de 75 escolas médicas brasileiras ${ }^{10}$ e no estudo com 616 estudantes de Medicina da Universidade de São Paulo ${ }^{11}$.

O presente estudo demonstrou que a presença de sonolência diurna em estudantes de Medicina está associada a menores escores gerais de habilidades sociais. Essa associação entre distúrbios do sono e habilidades emocionais tem sido apontada por outros estudos ${ }^{31,32}$. A presença de sonolência excessiva associa-se a maior dificuldade de leitura de ambiente, ou seja, menor capacidade de identificar as variáveis do ambiente para reagir de acordo com a situação. Isto explica o menor desempenho na habilidade de autoexposição a desconhecidos e situações novas (Fator 4) entre os estudantes sonolentos. Uma situação nova ou desconhecida exige maior atenção do indivíduo, o que se torna uma dificuldade para uma pessoa sonolenta ${ }^{14}$.

As habilidades sociais podem ser consideradas um elo não só entre o indivíduo e seu ambiente, mas também entre o indivíduo e seu semelhante, que no momento da prática profissional se coloca como a pessoa atendida ${ }^{2,14,30}$.

Esta pesquisa traz um modelo original para avaliação de competências gerais esperadas em um profissional da saúde, uma vez que analisa a presença de habilidades sociais e a sonolência diurna em estudantes de Medicina. No entanto, o estudo tem limitações por utilizar uma amostra de conveniência e se restringir ao período pré-internato, escopo deste estudo. Ainda, o desenho transversal do estudo não permite conclusões de causa e efeito entre as variáveis estudadas (habilidades sociais e sonolência diurna), somente de associação entre elas. Porém, tendo em vista sua originalidade e resultados, os autores acreditam que o estudo traz contribuições importantes para reflexão da educação médica, principalmente no que se refere aos quatro primeiros anos do curso.

\section{CONCLUSÃO}

Existe um número significativo de estudantes de Medicina que apresenta baixo desempenho em habilidades sociais. Os escores de habilidades sociais não variam nos diferentes anos do curso e entre os sexos, mas estão associados à presença de sonolência diurna excessiva.

$\mathrm{O}$ desenvolvimento das habilidades sociais garante o aprimoramento das relações pessoais e profissionais, contribuindo para a formação integral do médico e uma prática médica mais competente e humana. $\mathrm{O}$ tema abordado neste estudo pede atenção das escolas no sentido de incluir em seus currículos conteúdos e atividades de ensino voltados ao desenvolvimento de habilidades sociais.

\section{REFERÊNCIAS}

1. Almeida M. (org.) Diretrizes Curriculares Nacionais para os Cursos Universitários de graduação na área de saúde: Londrina: Rede Unida, 2003.

2. Caballo VE. Manual para Tratamento Cognitivo-Comportamental dos Transtornos Psicológicos da Atualidade: São Paulo: Editora Santos, 2006.

3. Del Prette A, Del Prette ZAP. Psicologia das Relações Interpessoais Vivências para o Trabalho em Grupo: Petrópolis: Vozes, 2007.

4. Tempski-Fiedler PZ. Avaliação da qualidade de vida do estudante de medicina e da influência exercida pela formação acadêmica. 308f. Tese (Doutorado) do curso de Pós-Graduação da Faculdade de Medicina da Universidade de São Paulo, São Paulo, 2008.

5. Paro HBM, Morales NM, Silva CHM, Rezende CHA, Pinto R, Morales R, Mendonça T, Prado M. Health-related quality of life of medical students. Medical Education 2010; 44: 227-235.

6. Baldassim S, Martins LN e Andrade AG. Traços de ansiedade entre estudantes de medicina. Arq Med ABC. 2006; 31(1): 27-31.

7. Wolf TM. Stress and Health: enharcing Well-being during medical school, Medical Education 1994; 28: 8-17.

8. Martins LAN. Atividade Médica: Fatores de risco para a saúde mental do médico. Rev. Bras. Clín. Terap. 1991; XX(9): 355-64

9. Millan LR, De Marco OLN, Rossi E, Arruda PCV. O Universo Psicológico do Futuro médico - Vocação, Vicissitudes e Perspectivas. São Paulo: Casa do Psicólogo; 1999.

10. Tempski P e Perotta B. Eu Quero, eu Preciso Dormir! Sonolência Diurna do Estudante de Medicina. Cadernos ABEM 2010; 6: 27-30

11. Alóe F, Pedroso A, Tavares SM. Epworth Sleepiness Scale Outcome in 616 Brazilian Medical Students. Arq. Neuropsiquiatr 1997; 55(2): 220-26.

12. Johns M. Reliability and Factor Analysis of the Epworth Sleepiness Scale. Sleep 1992; 15(4): 376-81. 
13. Rodrigues RN, Viegas CA, Silva AA, Tavares P. Daytime Sleepiness and Academic Performance in Medical Students. Arq Neuropsiquiatr 2002; 60(1): 6-11.

14. Del Prette ZAP, Del Prette A. Inventário de Habilidades Sociais: manual de aplicação, apuração e interpretação. São Paulo: Casa do Psicólogo, 2001.

15. Johns M. A New Method for Measuring Daytime Sleepiness: The Epworth Sleepiness Scale. Sleep 1991;14(6): 540-45.

16. Marins JJN, Rego S, Lampert JB, Araújo JGC. Educação Médica em Transformação. São Paulo: Hucitec, 2004.

17. Tempski P, Martins MA, Paro H. Teaching and Learning resilience: a new agenda in medical education. Medical Education. 2012; 46: 343-348

18. Machado $\mathrm{MH}$ (Org.). Os médicos no Brasil: um retrato da realidade. Rio de Janeiro: FIOCRUZ, 1997.

19. Montagner MI. Mulheres e trajetória na Faculdade de Ciências Médicas da UNICAMP: vozes singulares e imagens coletivas. Dissertação de Mestrado em Saúde Coletiva. Campinas: Faculdade de Ciências Médicas da Universidade Estadual de Campinas, 2007.

20. Scott J. Gênero: uma categoria útil para análise histórica. Educação e Realidade 1995; 20(2): 71-99.

21. Skinner BF. Ciência e Comportamento Humano. São Paulo: Brasiliense,1978.

22. Sidman M. Coerção e suas implicações. Campinas: Editorial Psy, 2003.

23. Del Prette, Z. A. P., Del Prette, A. Habilidades Sociais Cristãs: Desafios para uma Nova Sociedade. Petrópolis, RJ: Vozes, 2003.

24. Silva GSN. A construção do "ser médico" e a morte: significados e implicações para a humanização do cuidado. Tese (Doutorado) Faculdade de Medicina da Universidade de São Paulo, São Paulo, 2006.

25. Silvares EFM, Gongora MAN. Psicologia Clínica Comportamental: a inserção da entrevista com adultos e crianças. São Paulo: EDICON, 2006.
26. Freire P. Pedagogia da autonomia: saberes necessários à prática educativa. São Paulo: Paz e Terra, 1996.

27. Ministério da Saúde. Humaniza SUS - Clínica Ampliada e Compartilhada. Brasília: Editora MS, 2009.

28. Ristow AM. Formação Humanística do Médico na Sociedade do Século XXI: uma análise curricular. Setor de Educação Universidade Federal do Paraná, 2007.

29. Skinner BF. Questões recentes na análise do comportamento. Campinas: Papirus, 1991.

30. Del Prette ZAP, Del Prette A. Psicologia das Habilidades Sociais. Petrópolis: Vozes, 1999.

31. Rosen IM, Gimotty PA, Shea JA, Bellini LM. Evolution of sleep quantity, sleep deprivation, mood disturbances, empathy, and burnout among interns. Acad Med 2006; 81: 8285.

32. McNamara P, Pace-Schott EF, Johnson P, Harris E, Auerbach $\mathrm{S}$. Sleep architecture and sleep-related mentation in securely and insecurely attached people. Attach Hum Dev 2011; 13(2): 141-154.

\section{CONTRIBUIÇÃO DOS AUTORES}

Maria Cristina Kloster, Bruno Perotta, Alfredo Hauer Jr e Patricia Zen Tempski conceberam o estudo, coletaram e analisaram os dados, preparam e revisaram o manuscrito. Helena Paro analisou os dados, preparou e revisou o manuscrito. Todos os autores aprovaram a versão final do manuscrito encaminhada para publicação.

\section{CONFLITO DE INTERESSES}

Declarou não haver.

\section{ENDEREÇO PARA CORRESPONDÊNCIA}

Maria Cristina Kloster

E-mail: cristinakloster@gmail.com 